\title{
Intravital imaging of intestinal lacteals unveils lipid drainage through contractility
}

\author{
Kibaek Choe, ${ }^{1}$ Jeon Yeob Jang,,${ }^{2,3}$ Intae Park, ${ }^{2}$ Yeseul Kim, ${ }^{1}$ Soyeon Ahn, ${ }^{1}$ Dae-Young Park, ${ }^{2}$ Young-Kwon Hong, ${ }^{4}$ Kari Alitalo, ${ }^{5}$ \\ Gou Young Koh, ${ }^{2,6}$ and Pilhan Kim ${ }^{1,2}$ \\ ${ }^{1}$ Graduate School of Nanoscience and Technology and ${ }^{2}$ Graduate School of Medical Science and Engineering, Korea Advanced Institute of Science and Technology, Daejeon, South Korea. ${ }^{3}$ Biomedical Research \\ Institute and Department of Otorhinolaryngology - Head and Neck Surgery, Pusan National University Hospital, Pusan National University School of Medicine, Busan, South Korea. ${ }^{4}$ Department of Surgery \\ and Department of Biochemistry and Molecular Biology, Norris Comprehensive Cancer Center, Keck School of Medicine, University of Southern California, Los Angeles, California, USA. ${ }^{5}$ Translational Cancer \\ Biology Program and Wihuri Research Institute, Biomedicum Helsinki, University of Helsinki, Helsinki, Finland. ${ }^{6}$ Center for Vascular Research, Institute for Basic Science, Daejeon, South Korea.
}

\begin{abstract}
Lacteals are lymphatic vessels located at the center of each intestinal villus and provide essential transport routes for lipids and other lipophilic molecules. However, it is unclear how absorbed molecules are transported through the lacteal. Here, we used reporter mice that express GFP under the control of the lymphatic-specific promoter Prox1 and a custom-built confocal microscope and performed intravital real-time visualization of the absorption and transport dynamics of fluorescence-tagged fatty acids (FAs) and various exogenous molecules in the intestinal villi in vivo. These analyses clearly revealed transepithelial absorption of these molecules via enterocytes, diffusive distribution over the lamina propria, and subsequent transport through lacteals. Moreover, we observed active contraction of lacteals, which seemed to be directly involved in dietary lipid drainage. Our analysis revealed that the smooth muscles that surround each lacteal are responsible for contractile dynamics and that lacteal contraction is ultimately controlled by the autonomic nervous system. These results indicate that the lacteal is a unique organ-specific lymphatic system and does not merely serve as a passive conduit but as an active pump that transports lipids. Collectively, using this efficient imaging method, we uncovered drainage of absorbed molecules in small intestinal villus lacteals and the involvement of lacteal contractibility.
\end{abstract}

\section{Introduction}

The small intestine is a major organ in which digestion of ingested foods and absorption of nutrients concomitantly and actively occurs. The luminal surface of the small intestine is densely covered with villi, which provide an extensive absorptive surface area. At the surface of each villus, enterocytes absorb the majority of digested and processed nutrients and materials, including drugs, across the apical membrane and release them into the lamina propria. The lacteal, located at the center of each villus, is an essential conduit for the drainage of absorbed lipid, while the mesenteric lymphatic vessels are the main conduits for the transport of drained lipids to the systemic circulation (1-3). In addition, orally ingested lipophilic drugs are assembled together with the ingested and digested lipids, and they are absorbed into enterocytes, drained, and transported through the lacteals and mesenteric lymphatic vessels and, ultimately, enter systemic circulation without passing the liver. This form of uptake is advantageous over common water-soluble drugs, because it can circumvent first-pass metabolism in the liver and enhance drug efficacy (4). In spite of the broad implications for metabolism, body homeostasis, and drug development, our knowledge of the dynamic mechanism of transepithelial lipid absorption

Authorship note: Kibaek Choe, Jeon Yeob Jang, and Intae Park contributed equally to this work and are co-first authors.

Conflict of interest: The authors have declared that no conflict of interest exists. Submitted: March 3, 2015; Accepted: August 27, 2015.

Reference information: / Clin Invest. 2015;125(11):4042-4052. doi:10.1172/JCI76509. across enterocytes and the subsequent transport via lacteals under in vivo conditions remains limited, mostly because of the lack of appropriate experimental tools (5).

Ex vivo methods, such as immunohistochemistry and transmission electron microscopy using fixed tissues, provide only single time point images of collapsed lacteals by fixation (6). In vitro models $(7,8)$ with cultured cell monolayers, such as Caco- 2 cells and lymphatic endothelial cells, have been used to investigate lipid or drug transport via lacteals and have provided some information about active absorption and permeability of lipids compared with other molecules, such as dextran. However, Caco-2 cells, which are derived from colorectal carcinoma, fail to mimic enterocytes, the natural small intestinal epithelial cells (7). More importantly, in vitro models inevitably possess artificial conditions that are quite different from natural physiological conditions in the lamina propria of villi. Most in vivo methods use lymph (9, 10) or vein (11) fistula from rats for collecting lipids assembled by enterocytes or a perfusion model (12) for the intestinal permeability test, and these in vivo methods have provided only an indirect view of lipid absorption dynamics in the villi at the cellular level.

In the present work, we investigate the in vivo absorption and transport dynamics of lipids and various exogenous molecules through the lacteals in small intestinal villi by using lymphatic reporter mice (Prox1-GFP mice) and a custom-built video-rate laser scanning confocal microscope with a customized imaging chamber. Surprisingly, this dynamic in vivo imaging revealed spontaneous contractile movement of lacteals that appeared to 
A

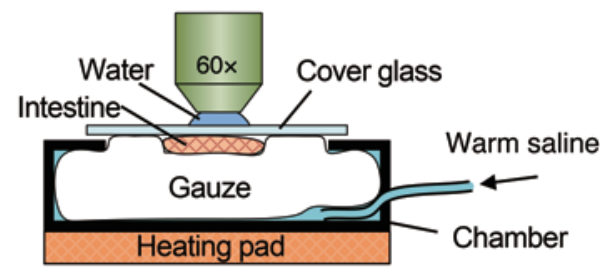

B

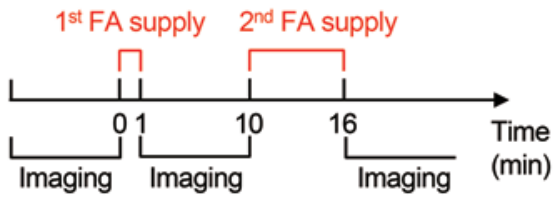

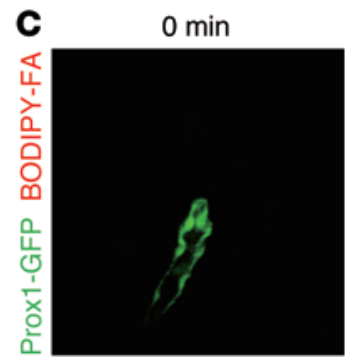
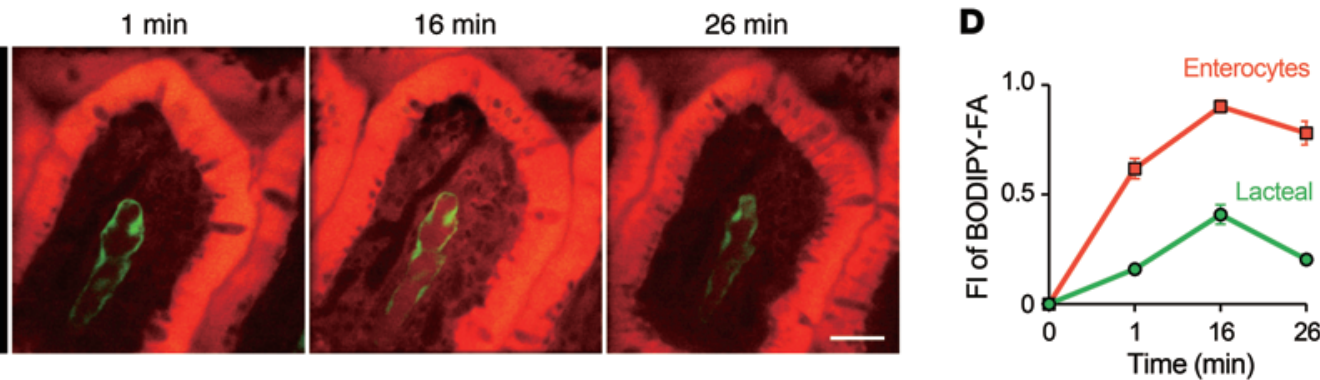

Figure 1. Intravital imaging to monitor FA absorption and clearing. (A) Schematic depiction of the intestine preparation, with the chamber for intravital imaging. (B) Schedule of FA supply and image acquisition. (C) Dynamic BODIPY-conjugated FA absorption and drainage in a single small intestinal villus. Scale bar: $30 \mu \mathrm{m}$. (D) Quantification of the average fluorescence intensity (FI) of BODIPY-conjugated FAs in enterocytes and lacteals. A total of 27 villi from 4 mice were analyzed. Error bars indicate SEM.

be mediated by smooth muscle contraction and controlled by the autonomic nervous system. Collectively, we uncovered efficient drainage of absorbed molecules in small intestinal villus lacteals, which act as active pumps through regulated contractibility.

\section{Results}

Visualization of fluorescent-tagged FA absorption and drainage in an individual villus. To investigate the lacteal function and transport dynamics of absorbed lipids within villi at the multicellular level in vivo, a custom-built intravital video-rate laser scanning confocal microscopy system $(13,14)$ and Prox1-GFP reporter mice $(15)$ were used (Supplemental Figure 1; supplemental material available online with this article; doi:10.1172/JCI76509DS1). The feasibility of using Prox1-GFP reporter mice to visualize lacteals in vivo was verified by immunohistochemistry staining (Supplemental Figure 2). The customized imaging chamber consisted of a detachable cover glass that can be temporarily removed from the intestinal lumen to allow free and rapid spontaneous drainage of FAs and then replaced during imaging to obtain a clear view of villi (Figure 1A and Supplemental Figure 1). Fully exploiting the potential of this imaging system, we supplied lipophilic fluorophore BODIPYconjugated FAs with a chain length of 12 (C12 FA) to the villi once and waited for 1 minute to observe the absorption into the enterocyte, and then FAs were supplied again in excess over the course of 6 minutes to clearly monitor the transport into the villi (Figure 1B). C12 FAs accumulated at a high rate in enterocytes after the first supply. After the second supply, the absorption of C12 FAs into the lacteal was more clearly visible, as was FA clearance from the inside of the villi over the following 10 minutes (Figure 1, C and D). In order to validate the actual absorption and drainage downstream of the lymphatics during the intravital imaging with our imaging chamber system, we visualized the transport of C12 FAs through the mesenteric collecting lymphatics with the same time schedule (Supplemental Figure 3). We observed that fluorescence intensity of C12 FAs in mesenteric collecting lymphatics and veins increased in accord with the supply of FAs on the exposed lumen and decreased as time went on, suggesting that there was indeed real absorption during intravital imaging of villi.

In addition, we investigated drainage of FAs with different chain lengths. More long-chain FAs (chain length of 14 to 16) are transported by the lymphatics compared with short-chain FAs (16-18), because long-chain FAs are selectively incorporated into lipoproteins, such as chylomicrons, which prefer to be transported by the lymphatics rather than by the blood vessels $(4,16)$. FAs with a chain length of 3 (C3 FAs) were both absorbed and cleared much more quickly than $\mathrm{C} 12 \mathrm{FAs}$, filling the lamina propria and the lacteal within 1 minute and being cleared away within 3 minutes, with minimal remaining FAs in the enterocytes (Supplemental Figure 4). Long-chain FAs with a chain length of 16 (C16 FA) showed similar absorption and drainage patterns in most villi compared with C12 FAs (Supplemental Figure 5, A and B). However, in some villi $(\sim 24 \%)$, delay of C16 FA release from the enterocytes was observed; the amount of FAs in both the lamina propria and the lacteals was increased at 10 minutes after the second supply (Supplemental Figure 5, C and D). On the other hand, this delay of FA release was not observed in C12 FAs. In addition, C16 FAs were cleared more slowly than C12 FAs in the lacteals (Supplemental Figure 5E). Collectively, these results suggest that the speeds of release from enterocytes and drainage inside villi for FAs are chain length dependent.

To investigate whether the system is applicable to neonates and if the neonates have different drainage speeds of the FAs compared with adults, we conducted the same experiments with early-age pups. We successfully visualized the FA drainage at postnatal days 0 and 20 and also observed that the C12 FAs were absorbed within 1 minute, which was more rapid than in adult mice (Supplemental Figure 6). Previous work led to the speculation that there is a specialized prelymphatic channel that mediates the transport of lipids through the lamina propria (6). However, in 
Table 1. Drainage of fluorescent molecules via intestinal villi

\begin{tabular}{|c|c|c|c|c|c|}
\hline \multirow[t]{2}{*}{ Molecules } & \multirow[t]{2}{*}{ MW } & \multirow{2}{*}{$\begin{array}{l}\text { Water solubility } \\
\qquad(\mathrm{mg} / \mathrm{ml})\end{array}$} & \multicolumn{3}{|c|}{ Detectable } \\
\hline & & & Enterocyte & LP & Lacteal \\
\hline BODIPY-conjugated C16 FA & $474 \mathrm{Da}$ & Low & 0 & 0 & 0 \\
\hline BODIPY-conjugated C12 FA & $472 \mathrm{Da}$ & Low & 0 & 0 & 0 \\
\hline BODIPY-conjugated C3 FA & $292 \mathrm{Da}$ & Low & 0 & 0 & 0 \\
\hline Methylene blue & $374 \mathrm{Da}$ & 50 & 0 & 0 & 0 \\
\hline Fluorescein & $376 \mathrm{Da}$ & 500 & 0 & 0 & 0 \\
\hline Rhodamine B & $479 \mathrm{Da}$ & 50 & 0 & 0 & 0 \\
\hline Evans blue & $961 \mathrm{Da}$ & 280 & $x$ & $x$ & $x$ \\
\hline TD & $10 \mathrm{kDa}$ & 50 & $x$ & $x$ & $x$ \\
\hline FITC & $389 \mathrm{Da}$ & $<0.1$ & 0 & $X$ & $X$ \\
\hline Topotecan & $421 \mathrm{Da}$ & 1 & 0 & 0 & 0 \\
\hline
\end{tabular}

our study, no such specialized channel was observed between the enterocytes and lacteal, which is consistent with another report (10) that suggested diffusion as the responsible mechanism for lipid transport through the interstitial space of villi.

Visualization of various exogenous molecules with different drainage characteristics in villi. We also investigated the absorption dynamics of other exogenous molecules with various biochemical properties and MWs by administering them with the same protocol (Table 1). Among water-soluble dyes, methylene blue (MW $374 \mathrm{Da}$ ), fluorescein (MW $376 \mathrm{Da}$ ), and rhodamine B (MW 479 Da) were absorbed into the enterocytes and then drained through the lacteal, whereas Evans blue (MW $961 \mathrm{Da}$ ) and tetramethylrhodamine-dextran (TD, MW $10 \mathrm{kDa}$ ) could not even enter the enterocytes (Figure 2, A-E). These results are consistent with those of a previous report showing that bioavailability sharply decreases as MW increases beyond 500 to $700 \mathrm{Da}(19,20)$. Interestingly, however, the amount of methylene blue in the lamina propria and the lacteal was much lower than that of fluorescein or rhodamine $\mathrm{B}$, although its MW was lower than that of the others.

When we induced physical damage to the epithelial barrier of enterocytes with surgical tweezers, not only the originally impermeable 10-kDa TD but also 2-MDa TD succeeded in infiltrating the villi to drain into the lacteals (Figure 3, A and B). Furthermore, $5 \%$ dextran sodium sulfate-induced (DSS-induced) ileitis increased the permeability of 10-kDa TD in ileac villi (Figure 3C). These results suggest that large molecules, up to at least $2 \mathrm{MDa}$, can invade the systemic circulation via lymphatic drainage when the villus epithelial barrier is damaged or impaired due to acute physical injury (e.g., undigested fish bone) or severe inflammation (e.g., irritable bowel syndrome).

In addition, we observed that a lipophilic dye, FITC (MW 389 $\mathrm{Da}$ ), was successfully absorbed into the enterocytes very rapidly, within 1 minute. Interestingly, however, the dye molecules were not released through the basolateral membrane of the enterocytes into the lamina propria, even though FITC's MW is lower than that of rhodamine B (Figure 2F). This result may be because the isothiocyanate-reactive group of FITC might bind amine and sulfhydryl groups on proteins inside the enterocytes. Finally, we also observed the absorption and drainage of topotecan, a chemotherapeutic agent for ovarian and lung cancers that is approved for oral use by the US Food and Drug Administration, into the lacteal (Figure 2G). Collectively, these results imply that both the absorption by the enterocytes and the release of absorbed substances into the lamina propria are a tightly regulated under a selective process, while lacteals are relatively permeable to a wider variety of molecules with larger sizes.

Real-time visualization and characterization of lacteal contraction. By virtue of the real-time cellular-level intravital imaging capability of the custom-built video-rate laser scanning confocal microscopy platform $(13,14)$, we made an unanticipated discovery of the contractile movement of lacteals. In addition to the periodic change in the diameter of lacteals, cells within the lacteals, identified as black spots that may be leukocytes, also moved repeatedly in concert with the lacteal contraction (Figure 4A and Supplemental Video 1). To further analyze this finding, three dynamic parameters were compared: lacteal contraction, cell velocity, and vertical villus motion in a direction parallel to that of cell motion (Figure 4, B-D). We found that, as the lacteal contracted and relaxed, the cell within subsequently moved down and up, respectively (Figure 4, D and E). Notably, the cell velocity was 100 times higher than the radial velocity of the lacteal wall (lacteal diameter change per second), which is consistent with a previous report regarding mesentery collecting lymphatics (21). This result supports the idea that lacteals can also contract to drive the fluid within, similarly to collecting lymphatics. In addition, the lacteal contraction (revealed by lacteal diameter measurement) and the vertical villus motion (revealed by villus displacement measurement) occurred simultaneously in a synchronized manner (Figure $4 \mathrm{~F}$ ). The velocity of the cell was 10 times higher than the vertical velocity of the villus (displacement per second) (Figure 4, C and G). Note that the cells that were observed inside the lacteals were only rarely observed during live imaging. Expanding our study, we examined the effect of lacteal contraction on FA drainage to investigate whether the speed of FA drainage was a direct consequence of lacteal contraction. The extent of FA clearance from the villi with low- and high-contractile lacteals was compared by imaging each twice at 0 and 10 minutes after the C12 FA supply. In the case of C16 FAs, we compared them by imaging at 10 and 20 minutes after the FA supply, due to the delay of C16 FA release from the enterocytes (Supplemental Figure $5, C$ and D). Here, we defined the lacteal contraction as the average lacteal diameter change $(\mu \mathrm{m})$ multiplied by the frequency of contraction $(\mathrm{Hz})$. The villi with highly contractile lacteals indeed displayed a faster FA clearance rate compared with that of the villi with less contractile lacteals $(P=0.06$ for C12, $P=0.01$ for C16) (Figure $4 \mathrm{H}$ ). This finding directly indicates that lacteal contraction facilitates efficient drainage of FAs. 

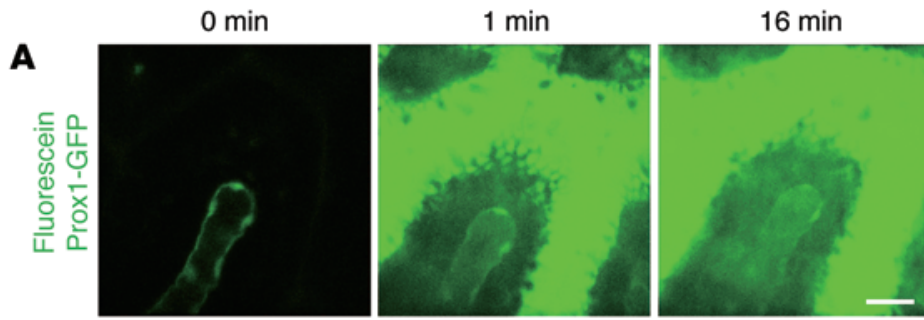

B
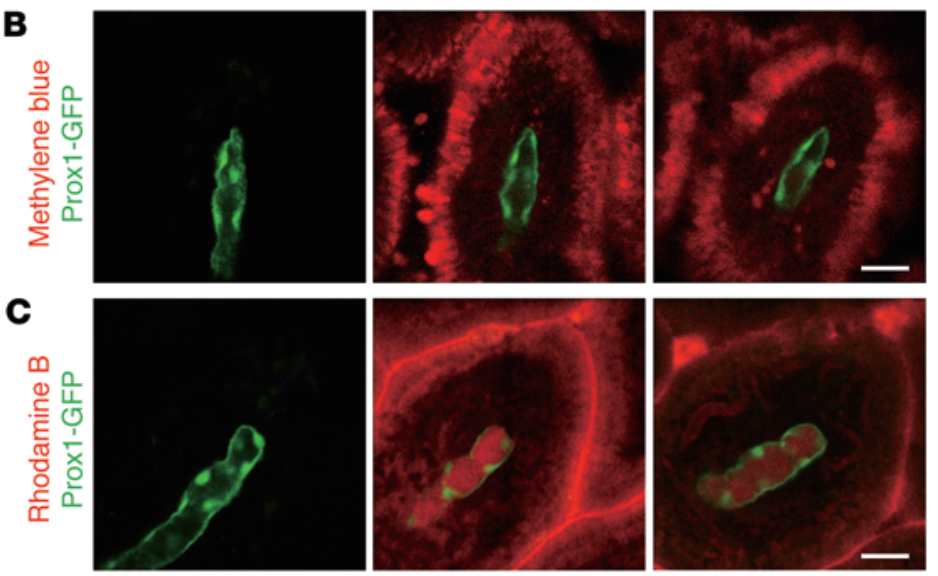

D
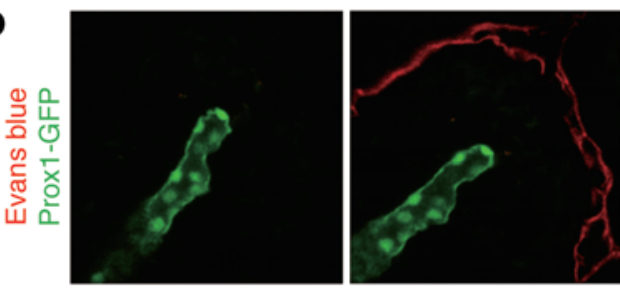

$\mathbf{E}$
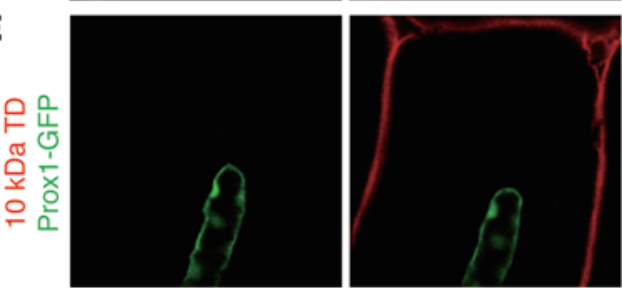

$\mathbf{F}$
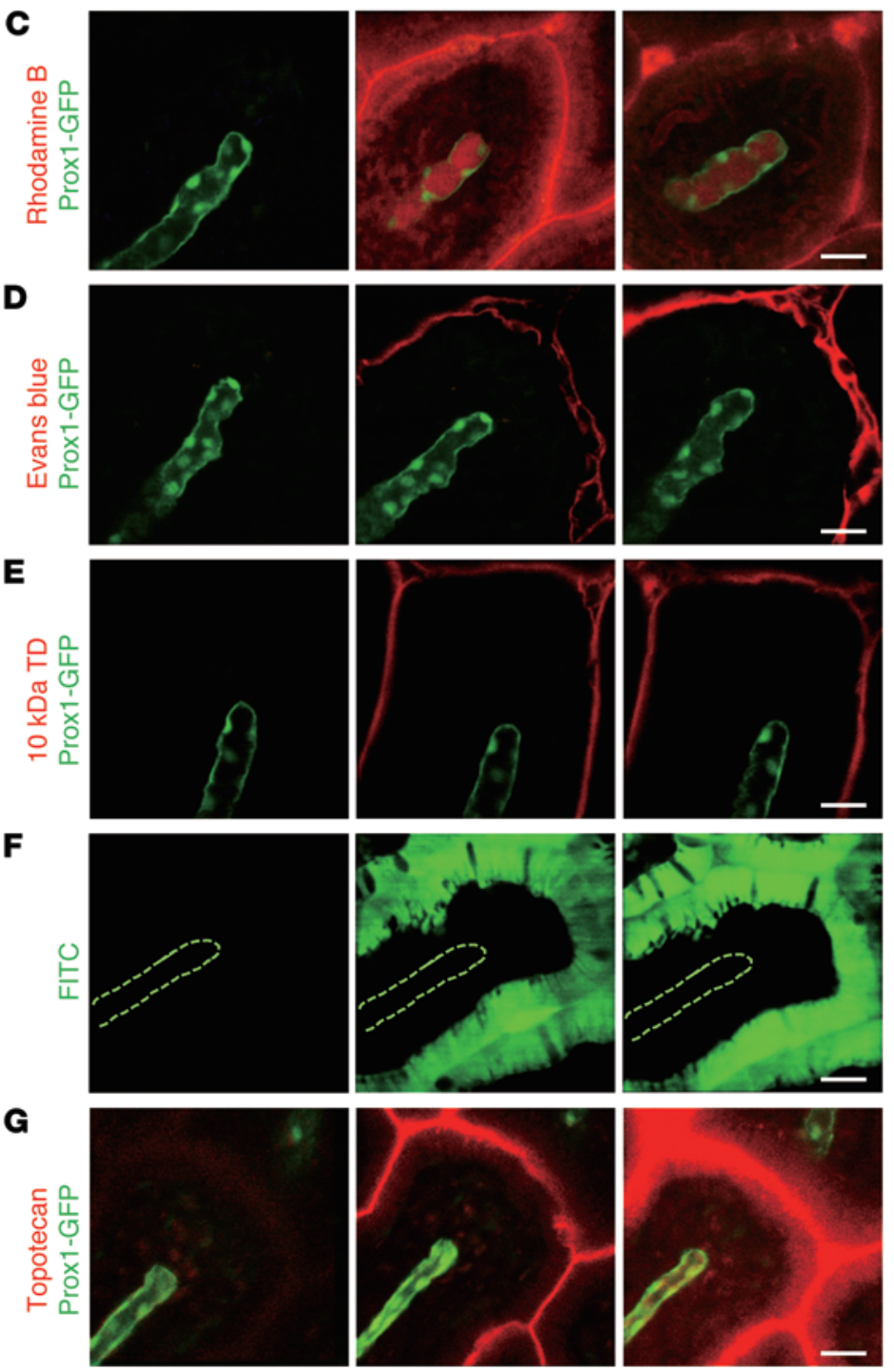

To explore how lacteals contract autonomously, we performed immunohistochemistry staining and confirmed the presence of smooth muscle cells (Supplemental Figure 7A). This confirmation is supported by a previous study (22) that showed the close proximity of muscle cells to lacteals by electron microscopic images of the lamina propria of villi, which suggested the presence of direct
Figure 2. Comparisons of the drainage of fluorescent dyes and drugs via small intestinal villi. Representative intravital images showing the drainage of the water-soluble dyes (A) fluorescein, (B) methylene blue, (C) rhodamine B, (D) Evans blue, and (E) 10-kDa TD. (F) Representative intravital images showing the drainage of lipophilic dye, FITC. The dotted lines delineate the lacteal. (C) Representative intravital images showing the drainage of an intrinsically fluorescent chemotherapeutic agent, topotecan. Representative images were each obtained from more than 3 independent experiments. Scale bar: $30 \mu \mathrm{m}$.

mechanical force acting on lacteals. To verify these speculations about smooth muscle-mediated lacteal contraction, we performed intravital imaging using actin-DsRed transgenic mice, in which we could identify longitudinally branching actin filaments, presumably smooth muscles, that matched those observed by immunohistochemistry staining (Supplemental Figure 7, A and B). From the intravital imaging, we revealed that the lacteals are surrounded by two or three branches of these actin filaments that pass over each other and contract periodically, inducing lacteal contraction (Supplemental Figure 7C and Supplemental Video 2). Importantly, we observed that the smooth muscle branches surrounding the lacteal moved in the opposite direction to the other smooth muscle branches of the villus (Supplemental Figure 8 and Supplemental Video 2), suggesting that the lacteal can contract independently of villus contraction. To clearly visualize lacteal and smooth muscles together, we generated a Prox1-GFP actin-DsRed double-transgenic mouse and found that actin filaments adjacent to the lacteal showed periodical movement, inducing lacteal contraction (Figure 5 and Supplemental Video 3).

Lacteal contraction is regulated by the autonomic nervous system. The autonomic nervous system regulates intestinal muscle contraction and absorption of nutrients and fluids $(23,24)$. Many types of smooth muscle cells, including those in the small intestine, retain subtypes of cholinergic and adrenergic receptors (25). Atropine, a representative antagonist of muscarinic acetylcholine receptor in the parasympathetic nervous system, hinders the mechanical activity of small intestine $(26,27)$. To test whether the parasympathetic nervous system also regulates lacteal contraction, we first applied acetylcholine $(200 \mu \mathrm{g}$ in $40 \mu \mathrm{l})$ and atropine $(20 \mu \mathrm{g}$ in $40 \mu \mathrm{l}$ ) onto the mesentery region. Two minutes after acetylcholine and atropine administration, the lacteals showed significantly increased $(P=0.02)$ and decreased $(P=0.008)$ lacteal contraction, respectively, compared with those that were administered PBS, which showed no significant difference (Figure 6, A, B, D, E, and G, and Supplemental Videos 4-6). We also tested for the effects of norepinephrine, which is released from sympathetic neurons and interacts with the $\alpha$ receptor of parasympathetic neurons, inhibiting acetylcholine release (26). It is known to directly induce smooth muscle relaxation by interacting with the $\beta$ receptor (26). Lacteal contraction decreased significantly $(P=0.002)$ when we introduced norepinephrine $(20 \mu \mathrm{g}$ in $40 \mu \mathrm{l})$ into the mesentery region, which supports our hypothesis (Figure 6, C, F, and G, and Supplemental 
A
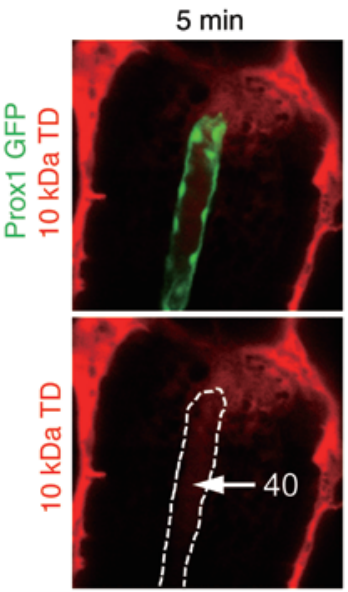

B
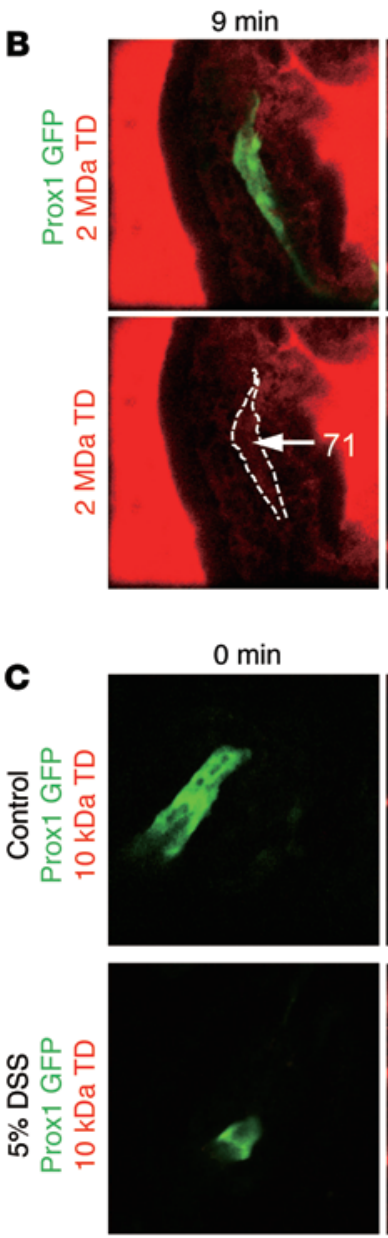

$7 \mathrm{~min}$
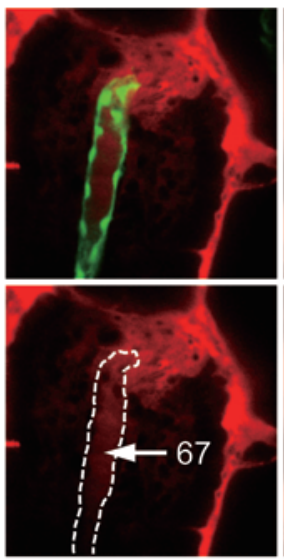

$14 \mathrm{~min}$

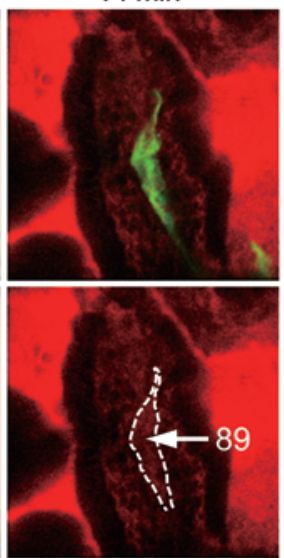

$1 \mathrm{~min}$
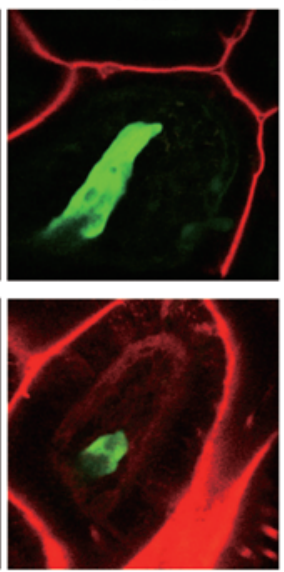

$10 \mathrm{~min}$
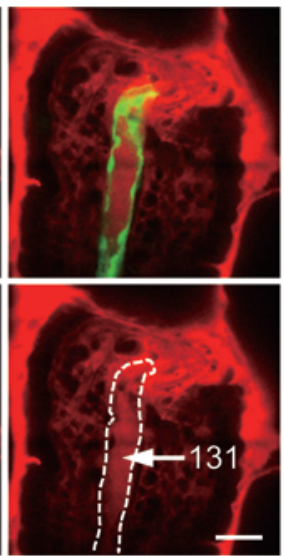

$19 \min$

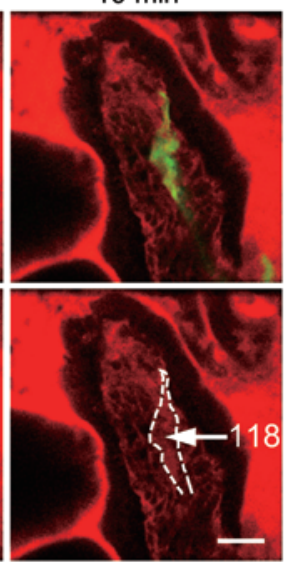

$16 \min$

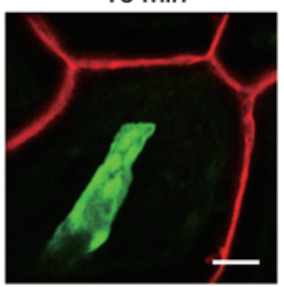

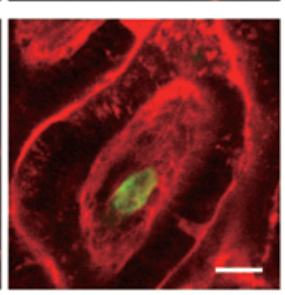

Figure 3. Large-molecule uptake in lacteals after physical and chemical damage of epithelial barrier. Time-lapse imaging of (A) $10-\mathrm{kDa}$ and (B) 2-MDa TD uptake in lacteals after physical damage to the epithelial barrier by surgical tweezers. The fluorescent intensity of TD (white numbers) increases inside the lacteals (white dotted lines) over time after damage. (c) Representative images showing the drainage of $10-\mathrm{kDa}$ TD in ileac villi of control mice and $5 \%$ DSS-induced inflammation model mice. Paracellular leakage of $10-\mathrm{kDa}$ TD was shown in the inflammation model mice. Representative images were each obtained from more than 3 independent experiments. Scale bar: $30 \mu \mathrm{m}$.

the ganglion-blocking agents mecamylamine $\left(3 \times 10^{-2} \mathrm{M}\right.$, $40 \mu \mathrm{l})$ and pentolinium $\left(6 \times 10^{-3} \mathrm{M}, 40 \mu \mathrm{l}\right)$ were administered onto the mesentery region, which included the intestinal wall ( $P=0.01$ and $P=0.002$, respectively) (Figure 6G). Interestingly, the contractile movement of the lacteals and the villus motion were not completely inhibited, whereas norepinephrine completely abrogated their movements (Figure 6F). Furthermore, even after we added an additional application of mecamylamine and pentolinium, each at a concentration 5 times greater than that used previously, at 15 minutes after the initial administration, the contractile movement of the lacteal and the villus motion were still not completely inhibited. Collectively, these results support the concept of enteric nervous control of lacteal contraction but also suggest the possibility of an additional regulation mechanism of lacteal contraction by the other receptormediated neurotransmission or nonneuronal control.

Additionally, we tested for the effect of blocking the neural signals of the ENS by tetrodotoxin (TTX) on the lacteal contraction. TTX is a potent neurotoxin that is known to bind to voltage-gated sodium channels of neurons to block action potential generation and propagation (29). However, TTX $\left(3 \times 10^{-7} \mathrm{M}\right)$ did not affect the frequency and magnitude of contractions of rabbit jejunum (30). Likewise, no significant difference in the lacteal contraction was observed after TTX $\left(6 \times 10^{-7} \mathrm{M}, 40 \mu \mathrm{l}, 40 \%\right.$ of $\mathrm{LD}_{50}$ for intravenous injection) was applied onto the mesentery region, including intestinal wall (Figure 6G). This result may be due to the blocking of all neural controls responsible for stimulating and inhibiting of smooth muscle contraction, while the autonomous contraction of smooth muscle will remain the same independently of neural signals (31). Therefore, we believe that the blocking of the neural signals of the ENS by TTX may not be able to change the lacteal contraction, Video 7). Specifically, atropine and norepinephrine significantly decreased both average amplitude and frequency of the lacteal contraction compared with PBS (Supplemental Figure 9).

We also investigated the effect of ganglion-blocking agents on the lacteal contraction. The ganglion-blocking agents block nicotinic acetylcholine receptor-mediated neurotransmission, which is the predominant mechanism of excitatory neurotransmission in the enteric nervous system (ENS) (28). Therefore, ganglion-blocking agents reduce smooth muscle motility in the intestine (26). Likewise, the lacteal contraction decreased when although TTX may induce other unknown defects in contractile movement and normal lacteal function.

Importantly, using the potent inhibitory effect of norepinephrine to our advantage, we acquired z-stack images of a contraction-inhibited lacteal, confirming that the observed changes of lacteal shape are not an artifact of the imaging focus drifting along the $z$ axis, but indeed are the result of bona fide lacteal contraction. Collectively, these data indicate that lacteal contraction is affected by the smooth muscle activity, which is regulated by the balance between the parasympathetic and sympathetic nervous 

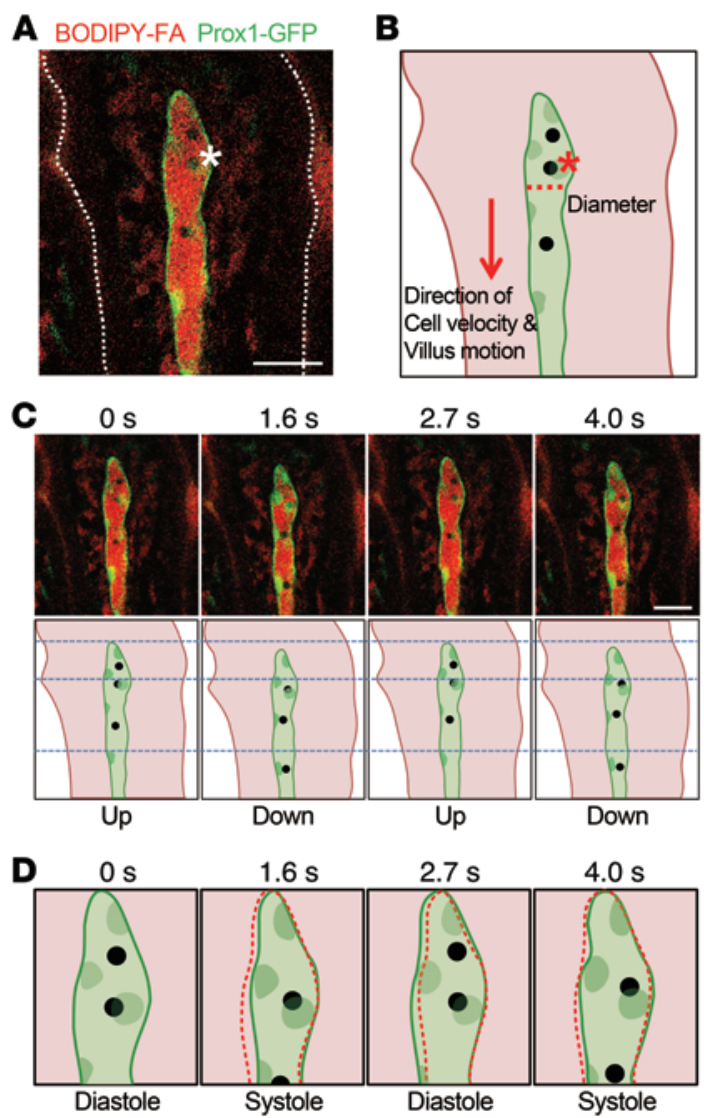

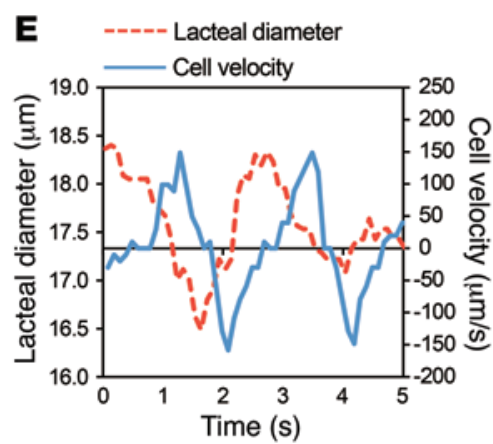

G

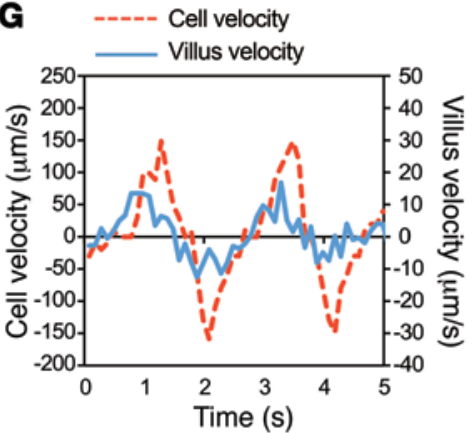

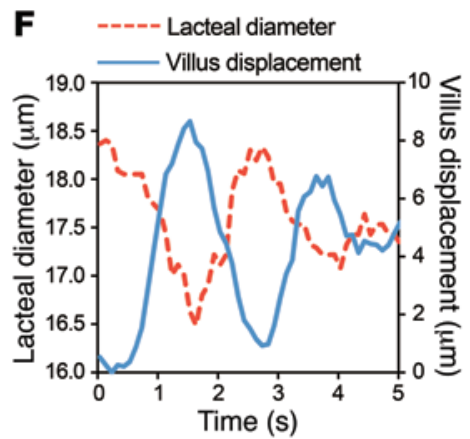

H

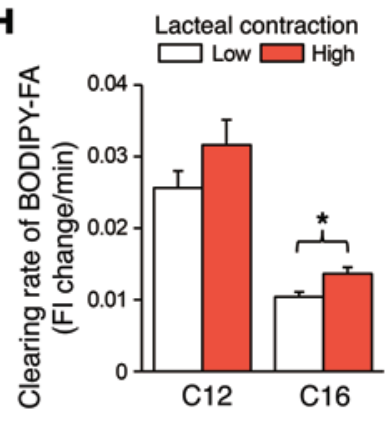

Figure 4. Contractile movement of lacteals. (A) Representative image and (B) illustration of a contracting lacteal. Asterisks indicate the same cell inside the lacteal. The white dotted line outlines the structure of the villus. The red dotted line indicates the point at which the lacteal diameter was measured. The red arrow indicates the positive direction used for cell velocity and villus motion measurement and analysis. Scale bar: 30 um. (C) Images and their illustrations were taken at different time points to characterize the cell and villus movement. Scale bar: $30 \mu \mathrm{m}$. (D) Illustrations of the tip of the lacteal to represent lacteal contraction. The red dotted lines outline the structure of lacteal at the previous time point. (E) Comparison of lacteal diameter and cell velocity versus time. (F) Comparison of lacteal diameter and villus displacement versus time. (G) Comparison of cell velocity and villus velocity versus time. (H) Comparison of the clearing rate of C12 or C16 FAs, which was quantified by calculating and comparing the change of average fluorescence intensity in the lamina propria of lowly contracting lacteals against highly contracting lacteals. Low and high contraction were defined as the average of two measurements of lacteal contraction being either lower or higher than $0.81 \mu \mathrm{m} \bullet \mathrm{Hz}$, respectively. A total of 36 villi from 4 mice and 36 villi from 3 mice were analyzed for C12 and C16 FAs, respectively. ${ }^{*} P<0.05$, paired Student's $t$ test. Error bars indicate SEM.

systems, although the neural control is not essential to induce the lacteal contractile movement.

\section{Discussion}

The drainage and transport of absorbed lipid is a unique organspecific function of the intestinal lymphatic system, consisting of lacteals and mesenteric lymphatic vessels, which strongly implies a distinct regulatory mechanism (1). Nevertheless, the pathophysiology of lacteals has been scarcely studied, while that of mesenteric lymphatic vessels is already fairly well known $(21,32,33)$. In the present study, our intravital imaging system enabled us to visualize and characterize the absorption and drainage of lipids and various molecules through enterocytes and lacteals in vivo, which was impossible using other previously reported methods, such as the in vitro permeability assay (7) and in vivo perfusion model (12). Taking advantage of our imaging system, we comprehensively evaluated the absorption and transport dynamics of fluorescence-tagged FAs with different chain lengths and water-soluble and lipid-soluble substances and drugs in the intestinal villi in real time, which consisted of transepithelial absorption via enterocytes, diffusive distribution over the lamina propria, and subsequent transport through lacteals. During the visualization of FAs and lacteals by vital imaging, we found that diffusion is the most responsible mechanism for lipid transport through the interstitial space of villi and that the speeds of release from the enterocytes and drainage inside villi for FAs are chain length dependent. Longchain FAs may stay longer in the enterocytes to be synthesized into triglycerides and assembled into lipoproteins, while shorter chain FAs diffuse across the enterocytes more simply (4). Delay of C16 FA release from the enterocytes suggests that C16 FAs participate more in the synthesis of lipoprotein compared with C12 FAs. In addition, the shorter chain FAs are more hydrophilic and smaller in size after being released from the enterocytes. Hence, they are more quickly cleared out though the blood capillaries, rather than through the lacteals, because absorbed molecules in the lamina propria first encounter the blood capillary plexus prior to the lac- 


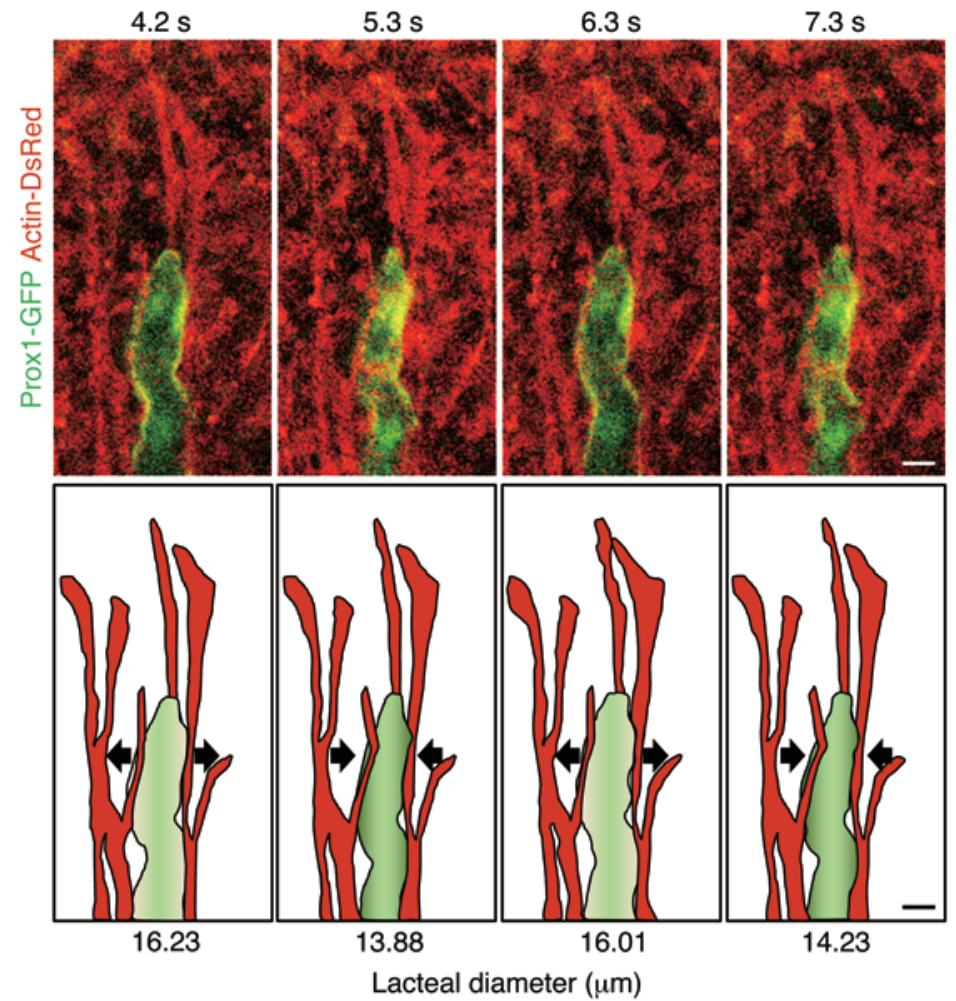

Figure 5. Lacteal contraction with adjacent smooth muscle. Serial video-rating images and their illustrations, showing the synchronous contractile movement of a lacteal (green) with actin fibers, which are assumed to be smooth muscles (red). Arrows indicate the direction of contractile movement. Representative images were obtained from more than 3 independent experiments. Scale bar: $10 \mu \mathrm{m}$.

by intricately interacting with surrounding smooth muscle movement, resulting in spontaneous contractibility under the regulations of the cholinergic and adrenergic nervous system, i.e., the autonomic nervous system. These findings define a unique role of lacteals in lipid transport as an active pump and provide critical mechanistic insight into how lacteals can drain a plentiful amount of dietary lipids.

Electrical vagal (parasympathetic) and periarterial (sympathetic) nerve stimulations increase and decrease the frequency of piston-like contraction of small intestinal villi, respectively (37). Likewise, we found that acetylcholine and norepinephrine increased and decreased the lacteal contraction. These results suggest that parasympathetic and sympathetic nerve stimulation can increase and decrease lacteal contraction, although autonomic nerve stimulation was not applied directly. It is also worthy to note that sympathetic nerve stimulation can decrease blood flow (37), which can change the intestinal villus teal located in the center of villus (Supplemental Figure 2), and blood flow is about 255 times faster than that of lymph flow (17).

Furthermore, by administering several exogenous molecules with varying biochemical properties and MW under the same protocol, we uncovered that absorption by the enterocytes and the release into the lamina propria are selectively regulated, while lacteals have much higher permeability. Importantly, our observation that lacteals were permeable to 2-MDa TD, which was too large to easily enter the blood capillaries, supports the idea that large-size molecules, such as chylomicrons, prefer to be transported by the lacteals rather than by the blood capillaries $(4,34)$. However, there are other factors for selective lacteal transport of chylomicrons. For example, Plagl2 $^{--}$mice showed impaired lacteal uptake of chylomicrons, which accumulated in the lamina propria of small intestinal villi but not in the lacteals $(5,35)$. This result suggests that there are unknown necessary processes for chylomicrons in the enterocytes for uptake into lacteals, since PlagL2 expression in small intestine is limited to the enterocytes $(5,35)$.

Most importantly, we discovered a spontaneous contractive property of lacteals. Previous reports only described villus motion as piston-like retraction and extension, which was speculated to be mediated by smooth muscles that are aligned longitudinally along the lacteal $(36,37)$ to facilitate the initial lymph drainage $(34)$. On the other hand, our study directly visualized the lateral contraction of lacteals in concert with adjacent smooth muscles, which showed that lacteals possess unique characteristics as a part of an organ-specific lymphatic system. As well as having the structural characteristics of initial lymphatic vessels, such as discontinuous button-like junctions and lack of pericyte coverage (38), we showed that lacteals exhibit mixed functions of initial and collecting lymphatics. Furthermore, they displayed enhanced absorptive ability motility (37) and can possibly affect the lacteal contraction. Furthermore, contraction of the mesenteric collecting lymphatics, which are the downstream lymphatics of the lacteals, may affect the lacteal contraction. Unfortunately, however, we could not exclude these factors in this study. Additionally, we investigated more about the role of the ENS in lacteal contraction by blocking nicotinic acetylcholine receptor-mediated neurotransmission of the ENS with ganglion-blocking agents and blocking all neural signal of the ENS with TTX. Ganglion-blocking agents, mecamylamine and pentolinium, decreased the lacteal contraction, supporting that the ENS can control the lacteal contraction, which is consistent with the effects of acetylcholine and norepinephrine on the lacteal contraction. Interestingly, mecamylamine and pentolinium significantly only decreased the average amplitude and the frequency of the lacteal diameter change, respectively (Supplemental Figure 9). The reason why they affected the contractile movement of lacteals differently remains unknown. Furthermore, our observation that TTX did not significantly affect lacteal contraction implies that the neural control of the ENS in lacteal contraction may not be essential. However, the frequency of the lacteal diameter change decreased by TTX compared with PBS (Supplemental Figure 9). Likewise, there might be unknown defects in the contractile movement of lacteals that are required to maintain normal lacteal function but have not yet been observed.

In the small intestine, digested lipids, free FAs, and monoglycerides are absorbed into enterocytes and subsequently resynthesized into triglycerides that are then incorporated into lipoproteins, such as very-low-density lipoproteins and chylomicrons $(4,39)$. The lipoproteins are then released into the lamina propria and tend to enter the circulation via lacteals. In this study, BODIPY-conjugated FA analogs were used to visualize absorption and drainage 
A
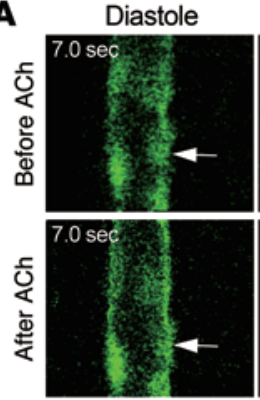

B
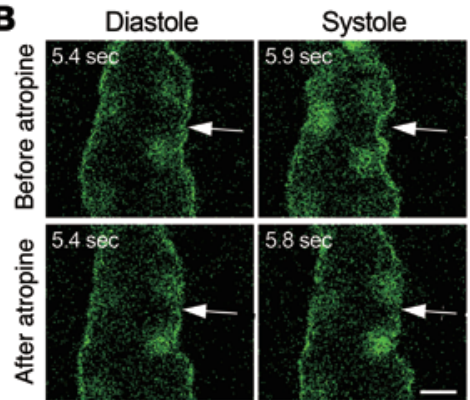

C

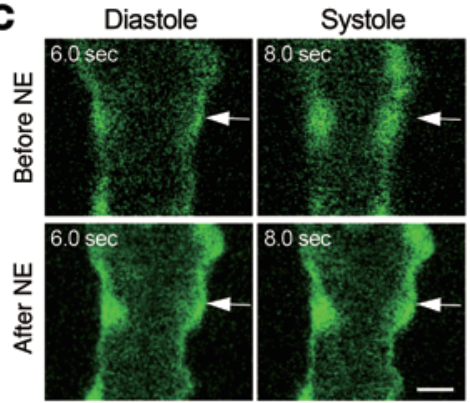
Systole
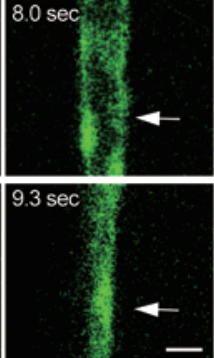

Systole

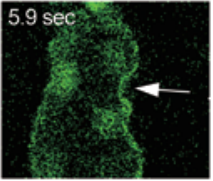

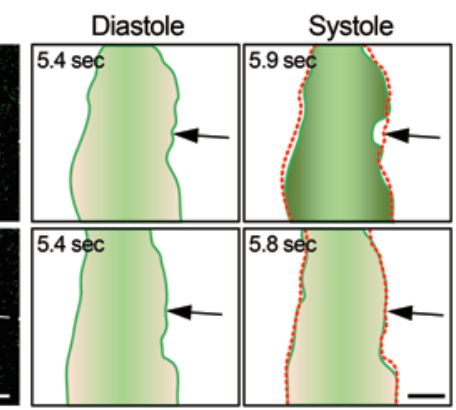
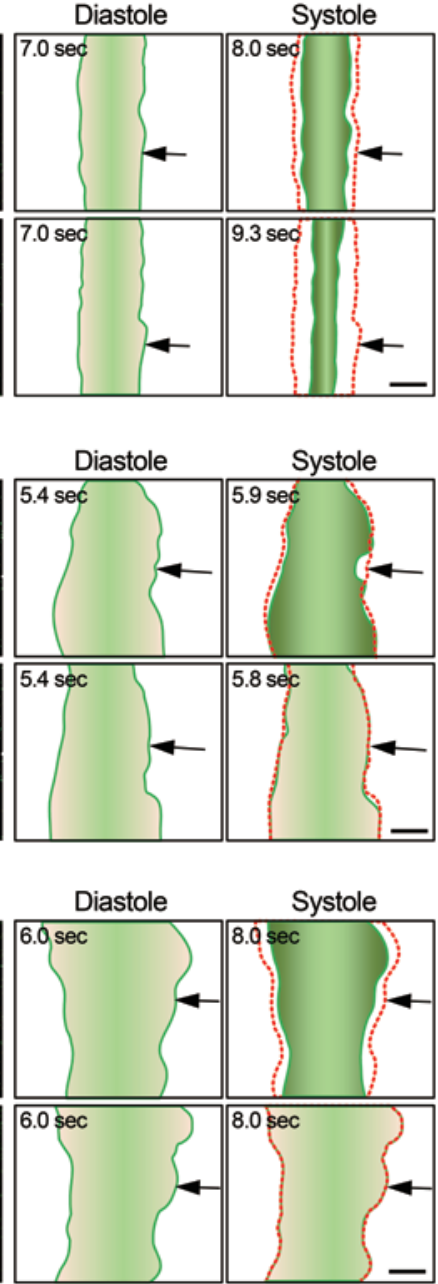

D

$\mathbf{F}$

E
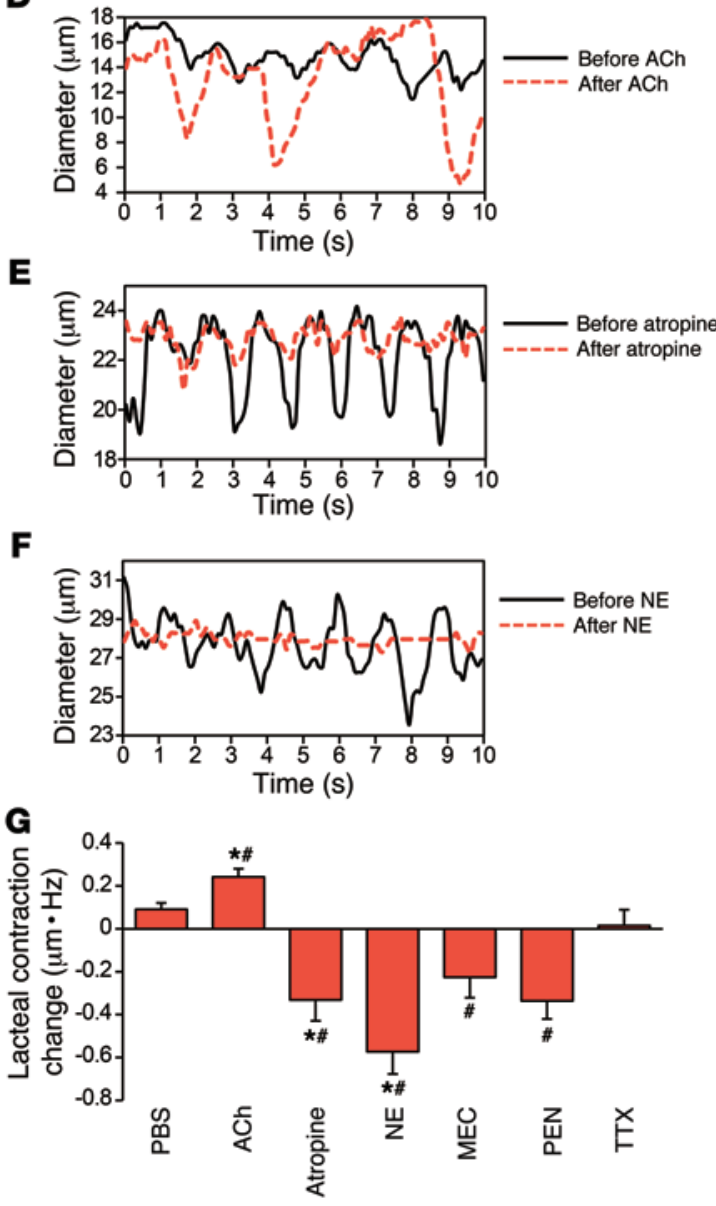

Figure 6. The autonomic neural control of lacteal contraction. Representative images of lacteal contraction and their illustrations before and after the administration of (A) acetylcholine (ACh), (B) atropine, and (C) norepinephrine (NE) in different lacteals. Arrows indicate the points at which the diameters were measured. The red dotted lines outline the structure of each lacteal at the initial time point. Scale bar: $10 \mu \mathrm{m}$. Lacteal diameter versus time, comparing the contractility of lacteals before and after the administration of (D) acetylcholine, (E) atropine, and (F) norepinephrine. (G) Quantification of the changes in lacteal contraction after administration of PBS, acetylcholine, atropine, norepinephrine, mecamylamine (MEC), pentolinium (PEN), and TTX, which were calculated by the average amplitude multiplied by the frequency of lacteal diameter change. The numbers of villi that were quantified are 37, 37, and 39 from 4 mice each for the PBS, acetylcholine, and MEC groups, respectively; 47 and 42 from 5 mice each for the atropine and norepinephrine groups, respectively; and 26 and 25 from 3 mice each for the PEN and TTX groups, respectively. ${ }^{*} P<0.05$, paired Student's $t$ test, differences between before and after drug administration. ${ }^{\#} P<0.05$, nonpaired Student's $t$ test, differences from the PBS group. Error bars indicate SEM.

of FAs with different chain length in small intestinal villi. BODIPYconjugated C16 FAs bind to albumin and FA-binding protein and are secreted from Caco- 2 cells as larger lipid particles, such as lipoprotein $(7,40)$. They have been used as a long-chain FA analog for observing lipid trafficking in vivo $(7,41)$. BODIPY-conjugated C12 FAs have been used to trace lipid droplets in adipocytes (42) and hepatocytes (43). They are incorporated into both glycerophospholipids and neutral lipids in lipid droplets in esterified forms (43). Hence, these BODIPY-conjugated FA analogs are reasonable lipid tracers. In addition, lipoproteins enhance the absorption of coadministered lipophilic drugs into the lacteals, minimizing firstpass metabolism $(4,44)$. However, although this study enabled in vivo tracking of fluorescent dye-tagged FAs in intestinal villi via lacteals for the first time to our knowledge, it is not yet possible to visualize the full synthesis process of coadministered lipids and lipophilic drugs and distinguish the concoction of the two substances from its constituents, mostly because of the lack of proper fluorescent labeling methods. Future technical developments that enable accurate characterization of the lipophilic drug assembly and absorption through the lacteal will serve as invaluable tools to achieve improved efficiency of lipophilic drug delivery.

In order to visualize small intestinal villi in vivo, a part of the intestinal loop was exteriorized and luminal surface was exposed and washed or wiped to remove fecal material as in previous studies $(45,46)$. However, a limitation exists with this method in that it could alter the normal physiological condition. Surgical opening of exteriorized intestinal loop could lead to inflammation and then affect the microenvironment of the villi and luminal microbiome. Minimal surgical opening with an endomicroscopy probe (13) can be an alternative approach to this limitation. Moreover, when we 
performed additional experiments on relatively empty parts of the intestinal loop without washing or wiping, we observed similar absorption and drainage patterns for C16 FAs in two independent experiments, although the fluorescence intensity was decreased in general compared with wiping method. However, there still may be unknown important alterations in lipid absorption due to mucus layer removal. Two-photon microscopy will help improve image quality by visualizing deep inside villi without removing fecal materials and mucus layer (47).

In conclusion, we successfully visualized the drainage pathways of lipids and various molecules through the intestinal villi in vivo by using Prox1-GFP reporter mice and our customized chamber system for intravital imaging. Furthermore, the finding of lacteal contraction gives critical insight into how lacteals efficiently drain large amounts of dietary lipids and fluids. The ability to dissect the absorption dynamics of intestinal villi at the cellular and molecular levels in vivo can provide new insight into the sophisticated mechanisms of transepithelial absorption and the subsequent transport of lipid molecules into the systemic circulation, and therefore, it will serve as a valuable tool to maximize the absorption efficacy of beneficial oral drugs and supplements.

\section{Methods}

Mice and materials. Prox1-GFP transgenic mice (15) were purchased from Mutant Mouse Regional Resource Centers and were maintained in a FVB/N-Crl:CD1(ICR) background. FVB/ N mice were purchased from Orient Bio. Actin-DsRed transgenic mice were purchased from The Jackson Laboratory and were maintained on a C57BL/6 background. Prox1-GFP actin-DsRed double-transgenic mice were generated by mating actin-DsRed mice with Prox1-GFP mice, which were backcrossed to the C57BL/6 background for at least 6 generations. All mice were bred in our SPF facility and fed with ad libitum access to standard diet (PMI Lab Diet) and water. BODIPY fluorescent-conjugated FAs (0.1 mg/ml, BODIPY FL C16, BODIPY 558/568 C12, and BODIPY FL C3, Invitrogen) and FITC $(0.1 \mathrm{mg} / \mathrm{ml}$, Sigma-Aldrich) were dissolved in $10 \%$ and $2.5 \%$ DMSO solution (Sigma-Aldrich), respectively. Methylene blue $(2.5 \mathrm{mg} / \mathrm{ml}$, Sigma-Aldrich), fluorescein (0.1 mg/ml, fluorescein sodium salt, Sigma-Aldrich), rhodamine B (0.1 mg/ml, Sigma-Aldrich), TD (2 mg/ml, 10 kDa; $10 \mathrm{mg} / \mathrm{ml}, 2 \mathrm{MDa}$, Invitrogen), Evans blue (0.2 $\mathrm{mg} / \mathrm{ml}$, Sigma-Aldrich), and topotecan hydrochloride $(0.1 \mathrm{mg} / \mathrm{ml}$, Sigma-Aldrich) were dissolved in PBS (Lonza). Acetylcholine chloride $(5 \mathrm{mg} / \mathrm{ml}$, Sigma-Aldrich), atropine (0.5 mg/ml, Sigma-Aldrich), norepinephrine $(0.5 \mathrm{mg} / \mathrm{ml}$, SigmaAldrich), mecamylamine hydrochloride (6.25 mg/ml, Sigma-Aldrich), and pentolinium tartrate $(3.125 \mathrm{mg} / \mathrm{ml}$, Sigma-Aldrich) were dissolved in PBS. TTX was dissolved in $0.1 \mathrm{M}$ citrate buffer $(0.5 \mathrm{mg} / \mathrm{ml})$ and diluted in PBS $(2 \mu \mathrm{g} / \mathrm{ml})$. For the acute ileitis mouse model, we added $5 \%$ (wt/vol) DSS ( 40,000 MW, Sigma-Aldrich) to drinking water for 5 days. To visualize the lacteals of wild-type mice, we injected 0.75 $\mathrm{mg} / \mathrm{kg}$ of anti-LYVE-1 antibody (MAB2125, clone 223322, R\&D Systems) conjugated with Alexa Fluor 647 (Invitrogen) at 12 hours before imaging. All mice were anesthetized by intramuscular injection (20 $\mathrm{mg} / \mathrm{kg}$ Zoletil [Virbac] and $11 \mathrm{mg} / \mathrm{kg}$ xylazine) before being sacrificed.

Intravital preparations. Before being administrated with the indicated agents for intravital imaging, the mice were deprived of food for 12 hours. The intravital imaging of the FA drainage in intestinal villi was performed after surgically opening the intestinal lumen. The level of anesthesia was continuously monitored during the experiments by using a toe pinch and maintained by intramuscular injection of half the dose of the initially injected Zoletil-xylazine mixture whenever a response was observed. The intravital imaging set-up for this study was modified from previously described methods $(45,46)$. About $8 \mathrm{~cm}$ of the proximal jejunum was exteriorized and placed in a chamber filled with wet gauze, of which about $1.5 \mathrm{~cm}$ was cut open along the antimesenteric border. The exposed intestinal lumen was carefully wiped with a saline-moistened swab to remove the debris in the lumen. For imaging of mice at postnatal day 0 , the wiping process was omitted, as their villi were easily damaged. Intravital imaging was performed over 3 sessions. The lacteal was first imaged before the first FA supply to observe the lacteal at resting state. Then, the FAs $(3 \mu \mathrm{g}$ in $30 \mu \mathrm{l})$ were supplied once before the second imaging session to observe the FA absorption into the enterocytes. Finally, the FAs were supplied in excess of 3 times with 2-minute intervals before the final imaging session so that we could observe the FA clearance through lacteals. In order to supply the FAs, the cover glass was temporarily removed from the intestines, and FAs were provided 1 and 6 minutes before the second and third imaging sessions, respectively, to facilitate rapid spontaneous drainage of FAs before mounting again with the cover glass. During FA supply, the exposed lumen was covered with a glass cap instead of a cover glass to avoid excessive exposure of villi. To keep the exteriorized jejunum moist, warm saline was supplied into the gauze in a chamber every 20 minutes. During the entire period of intravital imaging, the chamber temperature and the body temperature of the anesthetized mice were maintained at $37^{\circ} \mathrm{C}$ using a heating pad with temperature sensor and a body temperature regulator with rectal probe (PS-RT, Kent Scientific Corp.), respectively. In order to visualize mesenteric lymphatics and veins, 8-mm circular cover glass was placed on the mesentery region and $50 \mu \mathrm{l}$ Evans blue $(1 \mathrm{mg} / \mathrm{ml})$ was injected intravenously into Prox1GFP mice immediately before imaging.

Observation of lacteal contraction. To investigate the effect of lacteal contraction on the speed of FA drainage, the villi were imaged in real-time (30 frames per second) for 10 seconds immediately after the second supply of C12 FAs, and then the same villi were imaged again 10 minutes later. In case of C16 FAs, the villi were imaged 10 minutes after the second supply, and then the same villi were imaged again 10 minutes later. The lacteal contraction was defined as the average amplitude multiplied by the frequency of lacteal diameter change during the measurement time (10 seconds). Low and high contraction were defined as the average of two measurements of lacteal contraction being either lower or higher than $0.81 \mu \mathrm{m} \cdot \mathrm{Hz}$, respectively. In the experiment regarding the autonomic neural control of lacteal contraction, to increase the activity of the small intestines and therefore the lacteal motility, BODIPY-conjugated C12 FAs were dropped onto the opened lumen 50 minutes before administration of the drugs onto the mesentery region, and lacteals that were contracting vigorously before administration of the drugs were selected for study.

Video-rate intravital microscopy system. Intravital imaging of the intestinal villi was performed using a home-built video-rate microscope modified from a previously described confocal imaging platform $(13,14)$. Four continuous-wave lasers emitting at $405 \mathrm{~nm}$ and $488 \mathrm{~nm}$ (OBIS, Coherent), $561 \mathrm{~nm}$ (Jive, Cobolt), and $640 \mathrm{~nm}$ (Cube, Coherent) were used as excitation sources for fluorescence imaging. Fluorescence signals were simultaneously detected by 3 bandpass filters (FF01-525/50, FF01-600/37, FF01-685/40, Semrock) and 3 
photomultiplier tubes (R9110, Hamamatsu). The specifications of the wavelengths used to excite and detect fluorescent signals are as follows: topotecan was excited by a $405-\mathrm{nm}$ laser and detected with the bandpass filter FF01-525/50 (48); GFP, FITC, BODIPY C16 FA, and BODIPY-conjugated C3 FA were excited by a $488-\mathrm{nm}$ laser and detected with the bandpass filter FF01-525/50; TD and BODIPY-conjugated C12 FA were excited by a 561-nm laser and detected with the bandpass filter FF01-600/37; and Evans blue was excited by a 640$\mathrm{nm}$ laser and detected with the bandpass filter FF01-685/40. $z$-axis resolution of $4 \mu \mathrm{m}$ per section was acquired with $100-\mu \mathrm{m}$ pinhole. Images were displayed as $512 \times 512$ pixels per frame at a frame rate of $30 \mathrm{~Hz}$ and stored in a hard disk in real time by using a custom-written software and the Matrox Imaging Library (MIL9).

Image processing and data analysis. The signal-to-noise ratio was enhanced by averaging the noise over 90 frames after postprocessing the real-time images (30 frames per second) by removing the motion artifact generated from peristalsis with a custom-written MATLAB program. This motion correction program was also used for measuring villus motion and for making Supplemental Videos 2-7. The villus was exposed to the excitation laser light for only 3 seconds to obtain one image. When we took several images continuously for the same villus, there were no differences in their fluorescence intensity, implying that the reductions of fluorescence intensity were due to real flow of fluorescent dyes but not due to photobleaching. Image J was used to measure the average fluorescent intensity and lacteal diameter and to track the flowing cells in the lacteal lumen. The sampling rate for measuring the lacteal diameter and cell velocity was $10 \mathrm{~Hz}$. To measure the amplitude and frequency of lacteal contraction, first we manually tracked the boundary of lacteal with ImageJ and calculated diameter changes. Two-Hz low-pass filtering was applied to the raw tracking data by using OriginPro 8 (OriginLab Corp.) to remove any potential artifacts induced by the manual tracking. Then, local maximum and minimum values were extracted, and nonsignificant small peaks below $1 \mu \mathrm{m}$ were removed with a custom-written MATLAB program.

Immunohistochemical analysis. Tissues were harvested, fixed with $1 \%$ paraformaldehyde in PBS, dehydrated with $20 \%$ sucrose solution, and embedded in tissue-freezing medium (Leica) or paraffin (Leica). Sectioned tissues were blocked for 1 hour at room temperature with $5 \%$ goat (or donkey) serum (Jackson ImmunoResearch) in PBST $(0.3 \%$ Triton $\mathrm{X}-100$ in PBS). After blocking, the samples were incubated overnight at $4^{\circ} \mathrm{C}$ with one or more of the following primary antibodies: anti-LYVE-1 (rabbit polyclonal, AngioBio), anti-CD31 (hamster, clone
2H8, Millipore), or anti- $\alpha$ SMA (Cy3-conjugated, clone 1A4, SigmaAldrich). After several washes with PBST, sections were incubated for 2 hours at room temperature with one or more of the following secondary antibodies: Cy3- or FITC-conjugated anti-hamster IgG (Jackson ImmunoResearch) or Cy3- or FITC-conjugated anti-rabbit IgG (Jackson ImmunoResearch). Nuclei were stained with DAPI (Invitrogen). A Zeiss LSM 510 confocal microscope equipped with argon and heliumneon lasers (Carl Zeiss) was used to visualize the fluorescent signals and to obtain digital images.

Statistics. Values are presented as mean \pm SEM. Nonpaired 2-tailed Student's $t$ test was used for the comparison of FA drainage and the comparison of lacteal contraction changes after drug administration compared with those after PBS administration. Paired 2-tailed Student's $t$ test was used for the comparison of FA clearance rate between lowly and highly contractile lacteals and the comparison of lacteal contraction before and after administration of drugs. Statistical significance was set at $P<0.05$.

Study approval. Animal care and experimental procedures were performed with the approval of the Animal Care Committee of Korea Advanced Institute of Science and Technology (KA2013-12).

\section{Acknowledgments}

We thank Su-Jin Seo, Ki Yong Hong, Jin Sung Park, Yulia Gaeun Lee, Inwon Park, Gwang Hyeon Lee, and Hyunji Song for their assistance and discussions. This work was supported by grants from the National Research Foundation (NRF) (NRF-2013-036003, to G.Y. Koh), the World Class Institute program (WCI 2011-001, to P. Kim), the Engineering Research Centers program (NRF-20090083512, to P. Kim), the Global Frontier Project (NRF-M1AXA0022012M3A6A4054261, to P. Kim), the Converging Research Center Program (2011K000864, to P. Kim), and the Institute for Basic Science (IBS-R025-D1-2015, to G.Y. Koh) funded by the Ministry of Science, ICT and Future Planning (MSIP).

Address correspondence to: Pilhan Kim, Graduate School of Nanoscience and Technology, Korea Advanced Institute of Science and Technology, 291 Daehak-ro, Daejeon, 305-701, Republic of Korea. Phone: 82.42.350.1115; E-mail: pilhan.kim@kaist.ac.kr. Or to: Gou Young Koh, Center for Vascular Research, Institute for Basic Science, and Graduate School of Medical Science and Engineering, Korea Advanced Institute of Science and Technology, 291 Daehak-ro, Daejeon, 305-701, Republic of Korea. Phone: 82.42.350.2638; E-mail: gykoh@kaist.ac.kr.
1. Tammela T, Alitalo K. Lymphangiogenesis: molecular mechanisms and future promise. Cell. 2010;140(4):460-476.

2. Alitalo K. The lymphatic vasculature in disease. Nat Med. 2011;17(11):1371-1380.

3. Dixon JB. Mechanisms of chylomicron uptake into lacteals. Ann N Y Acad Sci. 2010; 1207(suppl 1):E52-E57.

4. Porter CJ, Trevaskis NL, Charman WN. Lipids and lipid-based formulations: optimizing the oral delivery of lipophilic drugs. Nat Rev Drug Discov. 2007;6(3):231-248.

5. Dixon JB. Lymphatic lipid transport: sewer or subway? Trends Endocrinol Metab.
2010;21(8):480-487.

6. Miller MJ, McDole JR, Newberry RD. Microanatomy of the intestinal lymphatic system. Ann N Y Acad Sci. 2010;1207(suppl 1):E21-E28.

7. Dixon JB, Raghunathan S, Swartz MA. A tissue-engineered model of the intestinal lacteal for evaluating lipid transport by lymphatics. Biotechnol Bioeng. 2009;103(6):1224-1235.

8. Heikkinen AT, Korjamo T, Monkkonen J. Modelling of drug disposition kinetics in in vitro intestinal absorption cell models. Basic Clin Pharmacol. 2010;106(3):180-188.

9. Hayashi H, Fujimoto K, Cardelli JA, Nutting DF, Bergstedt S, Tso P. Fat feeding increases size, but not number, of chylomicrons produced by small intestine. Am J Physiol. 1990; 259(5 pt 1):G709-G719.

10. Tso P, Balint JA. Formation and transport of chylomicrons by enterocytes to the lymphatics. Am J Physiol. 1986;250(6 pt 1):G715-G726.

11. Mansbach CM II, Dowell RF, Pritchett D. Portal transport of absorbed lipids in rats. Am J Physiol. 1991;261(3 pt 1):G530-G538.

12. Sadowski DC, Meddings JB. Luminal nutrients alter tight-junction permeability in the rat jejunum: an in vivo perfusion model. Can J Physiol Pharmacol.1993;71(10-11):835-839.

13. Kim P, et al. In vivo wide-area cellular imaging 
by side-view endomicroscopy. Nat Methods. 2010;7(4):303-305.

14. Choe K, Hwang Y, Seo H, Kim P. In vivo high spatiotemporal resolution visualization of circulating T lymphocytes in high endothelial venules of lymph nodes. J Biomed Opt. 2013;18(3):036005.

15. Choi I, et al. Visualization of lymphatic vessels by Prox1-promoter directed GFP reporter in a bacterial artificial chromosome-based transgenic mouse. Blood. 2011;117(1):362-365.

16. Chu SW, Hegsted DM. Lymph fatty acid composition during constant infusion of saturated fats of different chain lengths in the rat. J Nutr. 1980;110(11):2198-2206.

17. Kiyasu JY, Bloom B, Chaikoff IL. The portal transport of absorbed fatty acids. J Biol Chem. 1952;199(1):415-419.

18. Bloom B, Chaikoff IL, Reinhardt. Intestinal lymph as pathway for transport of absorbed fatty acids of different chain lengths. Am J Physiol. 1951;166(2):451-455.

19. Donovan MD, Flynn GL, Amidon GL. Absorption of polyethylene glycols 600 through 2000: the molecular weight dependence of gastrointestinal and nasal absorption. Pharm Res. 1990;7(8):863-868.

20. Goldberg M, Gomez-Orellana I. Challenges for the oral delivery of macromolecules. Nat Rev Drug Discov. 2003;2(4):289-295.

21. Dixon JB, Greiner ST, Gashev AA, Cote GL, Moore JE, Zawieja DC. Lymph flow, shear stress, and lymphocyte velocity in rat mesenteric prenodal lymphatics. Microcirculation. 2006;13(7):597-610.

22. Collan Y, Kalima TV. The lymphatic pump of the intestinal villus of the rat. Scand J Gastroenterol. 1970;5(3):187-196.

23. Widmaier EP, Raff $\mathrm{H}$, Strang KT, Vander AJ. Vander's Human Physiology: The Mechanisms Of Body Function. New York, New York, USA: McGraw-Hill; 2011.

24. Costa M, Brookes SJ, Hennig GW. Anatomy and physiology of the enteric nervous system. Gut. 2000;47(suppl 4):iv15-iv19.
25. Eglen RM, Reddy H, Watson N, Challiss RA. Muscarinic acetylcholine receptor subtypes in smooth muscle. Trends Pharmacol Sci. 1994;15(4):114-119.

26. Clark MA. Lippincott's Illustrated Reviews. Pharmacology. Baltimore, Maryland, USA: Wolters Kluwer Health/Lippincott Williams \& Wilkins; 2012.

27. Furness JB. The enteric nervous system and neurogastroenterology. Nat Rev Gastroenterol Hepatol. 2012;9(5):286-294.

28. Galligan JJ, North RA. Pharmacology and function of nicotinic acetylcholine and $\mathrm{P} 2 \mathrm{X}$ receptors in the enteric nervous system. Neurogastroent Motil. 2004;16(suppl 1):64-70.

29. Lee $\mathrm{CH}$, Ruben PC. Interaction between voltagegated sodium channels and the neurotoxin, tetrodotoxin. Channels (Austin). 2008;2(6):407-412.

30. Gershon MD. Effects of tetrodotoxin on innervated smooth muscle preparations. Br J Pharmacol Chemother. 1967;29(3):259-279.

31. Sanders KM, Koh SD, Ro S, Ward SM. Regulation of gastrointestinal motility - insights from smooth muscle biology. Nat Rev Gastroenterol Hepatol. 2012;9(11):633-645.

32. Muthuchamy M, Zawieja D. Molecular regulation of lymphatic contractility. Ann N Y Acad Sci. 2008;1131:89-99.

33. Davis MJ, Rahbar E, Gashev AA, Zawieja DC, Moore JE Jr. Determinants of valve gating in collecting lymphatic vessels from rat mesentery. $\mathrm{Am} J$ Physiol Heart Circ Physiol. 2011;301(1):H48-H60.

34. Kvietys PR, Granger DN. Role of intestinal lymphatics in interstitial volume regulation and transmucosal water transport. Ann N Y Acad Sci. 2010;1207(suppl 1):E29-E43.

35. Van Dyck F, et al. Loss of the PlagL2 transcription factor affects lacteal uptake of chylomicrons. Cell Metab. 2007;6(5):406-413.

36. Womack WA, Barrowman JA, Graham WH, Benoit JN, Kvietys PR, Granger DN. Quantitative assessment of villous motility. Am J Physiol. 1987;252(2 pt 1):G250-G256.

37. Womack WA, Mailman D, Kvietys PR, Granger DN. Neurohumoral control of villous motility.
Am J Physiol. 1988;255(2 pt 1):G162-G167.

38. Baluk P, et al. Functionally specialized junctions between endothelial cells of lymphatic vessels. JExp Med. 2007;204(10):2349-2362.

39. Hussain MM. A proposed model for the assembly of chylomicrons. Atherosclerosis. 2000;148(1):1-15.

40. Thumser AE, Storch J. Characterization of a BODIPY-labeled fluorescent fatty acid analogue. Binding to fatty acid-binding proteins, intracellular localization, and metabolism. Mol Cell Biochem. 2007;299(1-2):67-73.

41. Kassis T, et al. Dual-channel in-situ optical imaging system for quantifying lipid uptake and lymphatic pump function. J Biomed Opt. 2012;17(8):086005.

42. Targett-Adams P, et al. Live cell analysis and targeting of the lipid droplet-binding adipocyte differentiation-related protein. J Biol Chem. 2003;278(18):15998-16007.

43. Wang H, Wei E, Quiroga AD, Sun X, Touret N, Lehner R. Altered lipid droplet dynamics in hepatocytes lacking triacylglycerol hydrolase expression. Mol Biol Cell. 2010;21(12):1991-2000.

44. Harvey RA, Ferrier DR. Lippincott's Illustrated Reviews: Biochemistry. Philadelphia, Pennsylvania, USA: Wolters Kluwer Health; 2011.

45. Chieppa M, Rescigno M, Huang AYC, Germain RN. Dynamic imaging of dendritic cell extension into the small bowel lumen in response to epithelial cell TLR engagement. J Exp Med. 2006;203(13):2841-2852.

46. Zhu JB, Lee BG, Buhman KK, Cheng JX. A dynamic, cytoplasmic triacylglycerol pool in enterocytes revealed by ex vivo and in vivo coherent anti-Stokes Raman scattering imaging. J Lipid Res. 2009;50(6):1080-1089.

47. McDole JR, et al. Goblet cells deliver luminal antigen to $\mathrm{CD} 103(+)$ dendritic cells in the small intestine. Nature. 2012;483(7389):345-349.

48. Wiench B, Eichhorn T, Korn B, Paulsen M, Efferth T. Utilizing inherent fluorescence of therapeutics to analyze real-time uptake and multi-parametric effector kinetics. Methods. 2012;57(3):376-382. 